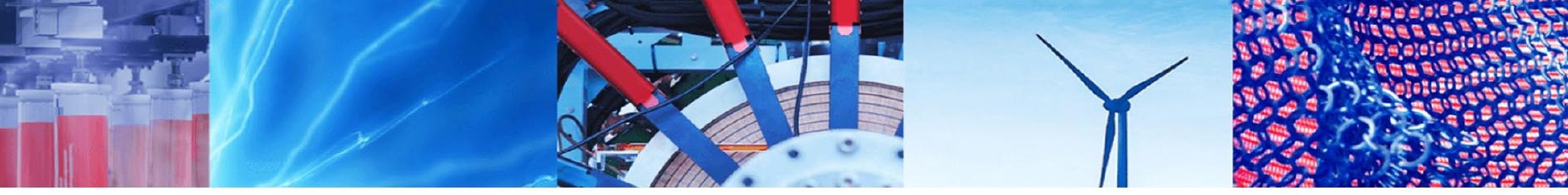

Review Paper

\title{
Reinforcement learning applied to games
}

\author{
João Crespo ${ }^{1}$. $\cdot$ Andreas Wichert ${ }^{1}$
}

Received: 23 November 2019 / Accepted: 17 March 2020 / Published online: 6 April 2020

(c) Springer Nature Switzerland AG 2020

\begin{abstract}
With the continuous evolution of computational power, especially in the computer graphics area, reinforcement learning has been gaining traction in the community as many novel methods are being created and older ones revamped. Many of these employ artificial neural networks, yet treat them as a black box system. In this paper, we start by introducing classic and recent developments in the area of machine learning, followed by an overview of the most relevant work done with deep neural networks applied to games. Our objective is to give the reader insights on how neural networks learn and clarify the decisions made in the development of modern systems.
\end{abstract}

Keywords Reinforcement learning · Neural network·Games

\section{Introduction}

Artificial intelligence (Al) systems have been a part of computer science for many years now and have been helping humans with multiple tasks ranging from medical diagnosis to self-driving cars. Most of the systems developed in the first decades of Al were algorithmic and made up of rules based on human expertise applied to algorithms such as minimax and alpha-beta pruning [16]. However, the goal has always been to develop an agent that could analyze, learn and act upon the environment on its own.

Machine learning is the area of artificial intelligence concerned with the development of models that use data or experience to learn how to succeed in a certain task. For instance, in [8] neural networks were used to analyze eye scans and detect signs of eye disease with a better accuracy than experts in the field. Moreover, in [49] an agent was trained to produce music based on previous examples made by humans. Games provide proof of concept in a sandbox-like environment that allows us to experiment and develop key ideas that might be useful in other areas. For instance, DeepMind used key aspects they learned while developing AlphaZero [36, 37], an agent capable of playing chess, go or shogi, to project AlphaFold's [27] neural network architecture. This system is capable of predicting the 3D shape of proteins, which can help scientists understand how to treat diseases they believe are caused by badly shaped proteins.

In this paper, we focus mainly in one type of machine learning called reinforcement learning and how it has been used to play various games. Firstly, we introduce some basic reinforcement learning concepts and algorithms designed throughout the past years. Secondly, we explore practical implementations of these algorithms and analyze some novel proposed algorithms that make use of the concepts from the first part, like AlphaGo [35], the first engine to beat the world champion in the game of Go, and proximal policy optimization [34]. With this work, we hope to gain better insights on how reinforcement learning systems work and how we can improve them.

The rest of the paper is organized as follows. Section 2 contains the background where we start by introducing basic machine learning concepts. The focus then shifts towards reinforcement learning and classical algorithms designed throughout the past years. Basic, convolutional and residual neural networks are also introduced in this

João Crespo, jpmcrespo@gmail.com | ${ }^{1}$ Instituto Superior Técnico, Lisbon, Portugal. 
section. Section 3 explores practical implementations of the aforementioned algorithms and analyzes some novel proposed ones that make use of concepts from the previous chapter, more specifically AlphaGo [35], the first engine to beat the world champion in the game of Go, AlphaZero [37], a more general approach aimed at zero-sum games, and proximal policy optimization [34]. Section 4 contains the conclusions and some insights into where these developments might be headed.

\section{Background}

\subsection{Types of machine learning}

Reinforcement learning (RL) is an area of machine learning concerned with mapping situations to actions, in order to maximize some idea of reward. One can make a comparison between this machine-based RL and the "conditional reflex" experiments Pavlov performed with dogs [25].

In reinforcement learning, the agent must discover which actions will yield the best rewards. It must have a perception of the state of its environment, predict and take actions that affect this state. One way of achieving this is by trial-and-error, making better predictions as the agent experiences more and more states and rewards.

As opposed to $R L$, in supervised learning the agent learns through examples provided by a supervisor. The examples are a set of attributes, called features, that describe a state, and a special feature, called the label, that describes which action the system should take when faced with that particular situation. The main objective of supervised learning algorithms is to be able to infer which combination of features should be mapped to which label (or category). The system can then take the appropriate action, even when the state was not present in the training set. This type of learning works very well in data problems, where the state is defined by a set of finite, well-defined features, for example as in [8]. However, in many real-world problems, namely interactive situations, it is not possible to obtain training sets that are representative of all the situations the agent might be faced with. For instance, in the game of chess, after only three moves by both opponents, 121 million board configurations are possible.

Unsupervised learning also differs from both reinforcement learning and supervised leanring. In this type of learning, the objective is to find structure hidden in unlabeled data.

\subsection{Reinforcement learning}

A reinforcement learning agent is usually forced to interact with the environment, even if it has no idea of what is going to happen and, in many cases, has to perform real-time action selection. This means that the agent cannot explore the world infinitely and exhaust all possible states and actions, as that would be unfeasible in most cases and defeat the purpose in others. One of the greatest challenges in RL is actions that affect not only the reward of the agent in the present state, but also in future ones. In Sect. 3, a few examples are explored.

Another great challenge we are faced with in reinforcement learning is finding a good trade-off between exploration and exploitation. To earn better rewards overall, an agent must follow actions that were previously attempted and which yielded better rewards. However, to find these actions an agent must attempt them without any previous experience and knowledge about them. It has to exploit actions it found to be most effective but also explore in order to discover such actions. This is especially hard when an action's effects are stochastic and the agent must choose an action many times to obtain a reliable estimate of the expected results. A strategy like adaptive epsilon-greedy [45] is often used, where the agent exploits the action it thinks is best with probability $1-\epsilon$ and explores another action with probability $\epsilon$.

There are a few key terms used in reinforcement learning that are worth mentioning:

- Policy Maps the agent's perceived states of the environment to the actions it should take when faced with said state, effectively dictating the agent's behavior. It may be a function or simply a matrix, and it can be stochastic, in the sense that the function returns the yields the probability of each action being chosen by the agent.

- Reward (or reward signal) The reward is a number sent by the environment to the agent that represents how good it was for the agent to perform the action in the state it was faced with. The agent's main objective is to maximize this reward in the long term. It is also how the agent knows whether it should change its policy or not. For instance, if an agent receives a low reward when selecting a particular action in some state, it might be better to change the policy in order to select another action when faced with the same situation.

- Value function The value function expresses how good a state is in the long run and can be viewed as the amount of reward an agent can yield in the future, going on from that state. Even if a particular state has a low reward signal, it might have a high value. For instance, in chess, sacrificing a queen might have a low reward, since the player is sacrificing his most powerful piece, but if it allows him to win the game shortly after, it should have a high value. This means that values are usually more important when making decisions, and a 
good estimate of these is crucial if we want the agent to perform well at a specific task.

- Model of the environment The model simulates the behavior of the environment and allows the agent to predict how the environment will react to a certain action. In model-based RL, an agent uses the model to plan which action it will take. However, a model is not necessary to perform reinforcement learning and model-free methods use trial-and-error to learn which actions should be taken.

\subsection{Temporal difference learning}

Definitely one of the greatest breakthroughs in reinforcement learning was the exploration of temporal difference (TD) methods by Sutton [40]. TD learning is a model-free technique in which an agent learns to predict a quantity based on other learned predictions, instead of waiting for the actual final outcome. TD algorithms are often used in reinforcement learning to predict the total amount of reward expected in the future.

Suppose an agent's objective is to predict some value in the future, for instance, whether the player playing as white will win in chess, or not, solely based on the board's state $s$ and the policy $\pi$ the agent is following. In more traditional (and supervised) methods, the prediction for that state at time step $t, V\left(s_{t}\right)$, would only be updated once the game was finished, most likely following a simple update like:

$V\left(s_{t}\right)=V\left(s_{t}\right)+\alpha\left[G_{t}-V\left(s_{t}\right)\right]$,

where $G_{t}$ is the actual outcome of the game, called the return, and $\alpha$ is the learning rate. The difference between the return and the actual estimate represents how far off the agent was to an accurate prediction. The learning rate is a value between 0 and 1 used to dictate how quickly the estimate should change with every new update. If this value is set too high, the estimate will be too sensitive, any outlier cases will have a big impact and the estimated value will vary widely. If set too low, the opposite happens and the estimate will change too slowly, increasing the time needed to reach a good evaluation.

\subsubsection{TD(0) and $\operatorname{TD}(\lambda)$}

One of the main problems with temporal difference learning lies in the fact that an agent only learns at the end of each episode. Sutton [40] proposes that the states in between time step $t$ and the final time step are also relevant to make predictions. The prediction problem described above can be easily extended to reinforcement learning agents with rewards in intermediate states, where instead of estimating the reward at the end of a sequence of episodes, the agent estimates the sum of the expected rewards found in the sequence. This means that at time $t+1$, one can immediately perform an update using the observed reward $R_{t+1}$ and the current estimate for the state at time stept $+1, V\left(s_{t+1}\right)$ :

$V\left(s_{t}\right)=V\left(s_{t}\right)+\alpha\left[R_{t+1}+\gamma V\left(s_{t+1}\right)-V\left(s_{t}\right)\right]$,

where $\alpha$ is the learning rate and $\gamma$ is the discount factor, used to effectively give more importance to rewards closer to the actual time step where the update is taking place. Both $\alpha$ and $\gamma$ are typically set between 0.9 and 1 . This method is called TD(0), or one-step TD because it only takes into account the reward of the next time step. We can, however, extend TD(0) to take into account more than one step when updating the estimates, which is called $n$-step TD. For instance, if we take into account two steps forward, our update would be based on the next two rewards the agent receives and the value for the state at the current time step $s_{t}$ would only be updated at $t+2$ by:

$G_{t: t+2}=R_{t+1}+\gamma R_{t+2}+\gamma^{2} V\left(s_{t+2}\right)$,

$V\left(s_{t}\right)=V\left(s_{t}\right)+\alpha\left[G_{t: t+2}-V\left(s_{t}\right)\right]$,

where now $\gamma^{2} V\left(s_{t+2}\right)$ is the discounted expected return for state $s_{t+2}$. Likewise, this can be extended to take into account an infinite number of steps, using the $n$-step return:

$G_{t: t+n}=R_{t+1}+\gamma R_{t+2}+\gamma^{2} R_{t+3}+\cdots+\gamma^{n} V\left(s_{t+n}\right)$,

$V\left(s_{t}\right)=V\left(s_{t}\right)+\alpha\left[G_{t: t+n}-V\left(s_{t}\right)\right]$.

This method uses $\left.V_{(} s_{t}\right)$ to compensate for the missing rewards beyond $R_{t+n}$. The number of steps $n$ considered can be any natural number. A valid update can also be made using multiple $n$-step rewards by weighing them. Reusing an example from Sutton's book [41], an update can be done using the average between the two-step and four-step return without losing the guaranteed convergence:

$G_{t}=\frac{1}{2} G_{t: t+2}+\frac{1}{2} G_{t: t+4}$.

Sutton's $\operatorname{TD}(\lambda)$ algorithm is only one way of averaging these returns. Each step's return is given a weight $\lambda^{n-1}$, where $\lambda$ is a value between 0 and 1 . The update is constructed using a return given by:

$G_{t}^{\lambda}=(1-\lambda) \sum_{n=1}^{T-t-1} \lambda^{n-1} G_{t: t+n}+\lambda^{T-t-1} G_{t^{\prime}}$

where $(1-\lambda)$ is a normalization factor. This return gives progressively less importance to rewards further away. When the agent reaches a terminal state, all subsequent 
$n$-step returns are equal to the sum of the rewards, $G_{t}$ and given whatever weight is left to distribute, $\lambda^{T-t-1}$. This generalized form can also perform the one-step update, if $\lambda=0$, and can also only account for the final actual sum, $G_{t}$ if $\lambda=1$ is chosen.

All these approaches are forward views, which means that the update is made by looking forward into subsequent states. However, forward views are relatively difficult to implement, since the updates depend on values that are not available at the time of the update. As a result, they need to be postponed until all the information is available.

Sutton [41] shows that there is a way of achieving nearly the same results with an algorithm that only looks at the TD error for the current time step and at the recently visited states, a backward view of $\operatorname{TD}(\lambda)$. Learning occurs at every time step, instead of being postponed to later, and can affect behavior in the next step, rather than $n$ steps later. In short, at each time step $t$ we update the value for the current state $s_{t}$ similarly to $\operatorname{TD}(0)$. As a result of having a better estimate for this state, we can perform another (partial) update to previous states. For instance, if we want to update the value for the state at time $t+2$, we would need to perform three updates:

$V\left(s_{t+2}\right)=V\left(s_{t+2}\right)+\alpha[\underbrace{R\left(s_{t+2}\right)+\gamma V\left(s_{t+2}\right)-V\left(s_{t+3}\right)}_{\delta_{t+2}}]$,

$V\left(s_{t+1}\right)=V\left(s_{t+1}\right)+\alpha \gamma \lambda \delta_{t+2}$

$V\left(s_{t}\right)=V\left(s_{t}\right)+\alpha \gamma^{2} \lambda^{2} \delta_{t+2}$

Although this works well when we can define every possible state the agent may be presented, in many cases this is not possible due to the sheer size of the state space. For the simple game of tic-tac-toe, there are almost 5500 possible legal states, and for bigger games this number quickly becomes astonishingly large. We can, however, eliminate the need to know all the states by creating a function $f$ with parameters $\theta$ that tries to approximate the value function $V$. Instead of learning the explicit value for every state, the agent's goal is to update $\theta$ to minimize the error between $f$ and $V$. There are many ways of doing this, and the most common approach is through gradient descent [30,31], using the error function $E$, which is typically the mean squared error:

$E=\frac{1}{2}\left(V\left(s_{t}\right)-f_{\theta}\left(s_{t}\right)\right)^{2}$

$V\left(s_{t}\right)=R\left(s_{t}\right)+f_{\theta}\left(s_{t+1}\right)$.

The parameters $\theta$ are then updated as follows. $\theta_{t+1}=\theta_{t}+\alpha \nabla_{\theta} E$

$\nabla_{\theta} E=\left(V\left(s_{t}\right)-f_{\theta}\left(s_{t}\right)\right) \cdot \nabla_{\theta} f_{\theta}\left(s_{t}\right)$,

where $\nabla_{\theta} E$ is the gradient of the error function with respect to $\theta$. It is also possible to adapt $\operatorname{TD}(\lambda)$ to multiple time steps, by taking into account not only the current time step's gradient, but also the ones from the previous updates, decayed progressively by $\lambda$ :

$\nabla_{\theta} E=\left(V\left(s_{t}\right)-f_{\theta}\left(s_{t}\right)\right) \cdot \sum_{k=1}^{t} \lambda^{t-k} \nabla_{\theta} f_{\theta}^{k}\left(s_{t}\right)$,

where $\nabla_{\theta} f_{\theta}^{k}\left(s_{t}\right)$ is the gradient at time step $k$.

\subsubsection{Q-learning}

One of the problems with the early temporal difference methods was that only the expected values for the provided policy $\pi$ were learned. However, in many cases, the optimal actions in each state are not known. Watkins [47] proposed a novel temporal difference control algorithm, known as Q-learning, and it is arguably the most famous reinforcement learning algorithm.

Q-learning is also a temporal difference learning method and maps every state-action pair $(s, a)$ to the expected discounted reward for the agent, instead of just the state, as seen previously in $\operatorname{TD}(\lambda)$. In this case, the learned value function $Q$ approximates the optimal value function $Q^{*}$ regardless of the policy that is being followed. The estimate for the state-action pair at time step $t,\left(s_{t}, a_{t}\right)$ is updated as:

$Q_{t+1}\left(s_{t}, a_{t}\right)=Q_{t}\left(s_{t}, a_{t}\right)+\alpha\left[R_{t}+\gamma \max _{a^{*} \in A} Q_{t}\left(s_{t+1}, a^{*}\right)-Q_{t}\left(s_{t}, a_{t}\right)\right]$,

where $\alpha$ is the learning rate, $R_{t}$ is the immediate reward, $\gamma$ is the discount factor, $s_{t+1}$ is the state in the next time step and $a^{*}$ is an action available in time step $t+1$. The action $a^{*}$ is selected based on the greedy policy. However, the actual action executed by the agent is dictated by a heuristic to balance exploration and exploitation, for instance the $\epsilon$ -greedy. This ensures that Q-learning converges to the optimal policy with enough time steps. This algorithm, similarly to $\operatorname{TD}(0)$, can be extended to take into account more than only one step into the future. The Q-learning is described in Algorithm 1. 


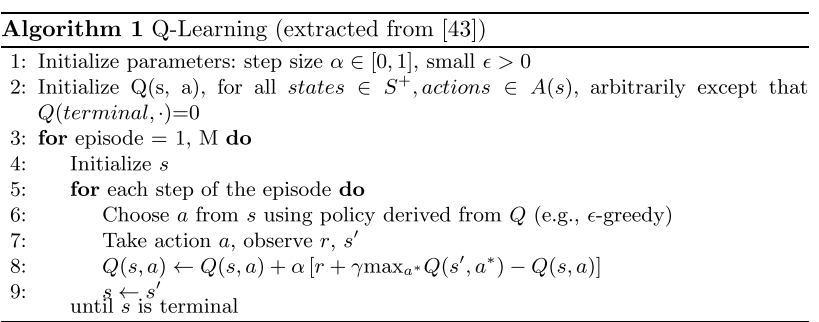

\subsection{Policy gradient methods}

While temporal difference learning methods are action value based, and as such, decide which action to take based on action value estimates, policy gradient methods learn and select an approximation for the policy and do not need a value function to decide which action to take. Value-based methods may also yield a bad policy, even if the approximation is good. Nevertheless, a value function can still be used to learn the policy.

This policy $\pi$ is a function with a set of parameters $\theta$ which can be, for example, the coefficients of a polynomial or the weights in a neural network. The real task is to find the parameters $\theta^{*}$ which make the policy prefer the action that yields the most reward for any given state, effectively attempting to maximize the objective function $\mathrm{J}$. For simplicity, only the discrete case is considered:

$J(\theta)=\mathbb{E}_{\pi_{\theta}}(r)=\sum_{s} P(s) \sum_{a} \pi_{\theta}(a \mid s) Q^{\pi_{\theta}}(s, a)$,

where $P(s)$ is the probability of state $s$ being the initial state. $\pi_{\theta}(a \mid s)$ is the probability of the agent choosing action $a$ when in state $s$ and $Q^{\pi_{\theta}}(s, a)$ is the long-term expected reward if the agent takes action $a$ in state $s$ and then follows policy $\pi_{\theta}$. We can attain $\theta^{*}$ using an optimization algorithm, and the most efficient ones are gradient methods [31], since these methods point in the direction of the greater reward. If we apply the gradient to the above expression, we obtain the following:

$$
\begin{aligned}
\nabla_{\theta} J(\theta) & =\nabla_{\theta} \sum_{s} P(s) \sum_{a} \pi_{\theta}(a \mid s) Q^{\pi_{\theta}}(s, a) \\
& =\sum_{s} P(s) \sum_{a} \nabla_{\theta}\left(\pi_{\theta}(a \mid s) Q^{\pi_{\theta}}(s, a)\right)
\end{aligned}
$$

which, as proven by Sutton in [42], can be unrolled into

$\nabla_{\theta} J(\theta)=\sum_{s} d^{\pi}(s) \sum_{a} \nabla_{\theta} \pi_{\theta}(a \mid s) Q^{\pi_{\theta}}(s, a)$,

where $d^{\pi}$ is a stationary distribution over all possible states $\chi$.

The function policy $\pi$ can be set as a known differential function, like the softmax, which allows us to use likelihood ratios from calculus to maximize the log of the policy instead of the actual policy, and the result will be the same. One advantage of this is converting multiplications inside the policy function into logarithmic additions, effectively simplifying the calculations of the derivatives. The resulting gradient is as follows:

$$
\begin{aligned}
\nabla_{\theta} J(\theta) & =\sum_{s} d^{\pi}(s) \sum_{a} \pi_{\theta}(a \mid s) \frac{\nabla_{\theta} \pi_{\theta}(a \mid s)}{\pi_{\theta}(a \mid s)} Q^{\pi_{\theta}}(s, a) \\
& =\sum_{s} d^{\pi}(s) \sum_{a} \pi_{\theta}(a \mid s) \nabla_{\theta} \log \left(\pi_{\theta}(a \mid s)\right) Q^{\pi_{\theta}}(s, a) \\
& =\mathbb{E}_{d, \pi_{\theta}}\left[\nabla_{\theta} \log \left(\pi_{\theta}(a \mid s)\right) Q^{\pi_{\theta}}(s, a)\right]
\end{aligned}
$$

The parameters are updated similarly to temporal difference learning by

$\theta=\theta+\alpha \nabla_{\theta} J(\theta)$.

Of course, this does not scale well for very large environments, since every time a parameter is updated the agent would have to run through all samples of the training data to calculate the expected value for the gradient. The REINFORCE algorithm [48] addresses this by taking a more practical approach and using stochastic gradient ascent. The REINFORCE algorithm is described as follows:

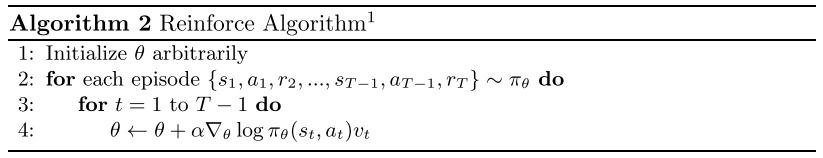

The agent starts by simulating an episode following the policy $\theta_{\pi}$ and then using the information gathered in each time step $t$ of the episode to update the parameters. $v_{t}$ is an unbiased sample of $Q^{\pi_{\theta}}\left(s_{t}, a_{t}\right)$, meaning that it is the actual reward gathered from $t$ until the end of the episode.

The main problem with the REINFORCE method is the high variance in the updates caused by the sampled rewards $v_{t}$. To solve this issue, actor-critic approaches [17] split the algorithm into two parts: one that maintains and runs the policy (actor) and another one that calculates an approximation of $Q$ (critic) using a value-based method, for instance TD(0).

\subsection{Artificial neural networks}

\subsubsection{Perceptron}

The perceptron is a system originally proposed by Rosenblatt in [28] for linear classification, based on the biological neuron model. It is an algorithm that combines a set of weights and features and uses an activation function to classify a given input $x$. The perceptron algorithm works in two steps. 

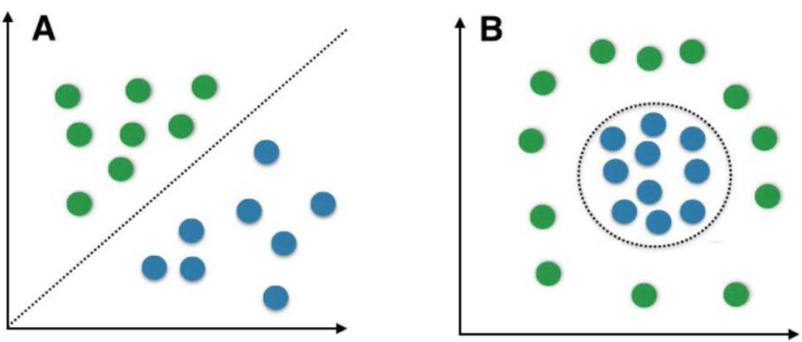

Fig. 1 Linear (a) versus non-linear data (b) (extracted from [26]). Random samples from two different classes are shown as colored spheres. The dotted line indicates the class boundaries that classifiers try to find. The perceptron would not be able to distinguish samples from $\mathbf{b}$

Firstly, it calculates the weighted sum net by multiplying each feature $x_{i}$ by its corresponding weight $w_{i}$ :

net $=w_{0}+w_{1} x_{1}+w_{2} x_{2}+\cdots+w_{n} x_{n}$.

Secondly, the algorithm feeds the result net into the neuron's activation function $f$, which produces the neuron's output $\sigma$, by mapping the input to values between 0 and 1 or -1 and 1, or any other range, depending on the function used. We can use any activation function, for example the sigmoid, as follows:

$\sigma=f(n e t)=\frac{1}{1+e^{-n e t}}$.

The purpose of the weights is to represent the strength of a node, or feature. These weights can be learned via training data and are updated using:

$w_{i}(t+1)=w_{i}(t)+\alpha(t-\sigma) x_{i}$

where $\alpha$ is the learning rate, $w_{i}(t)$ and $w_{i}(t+1)$ are, respectively, the current and the updated weights for feature $i, t$ is the expected output and $x_{i}$ is the actual value for feature $i$.

The activation function may be linear or non-linear, although the latter is much more commonly used. However, by computing the decision boundary, we can see that it is always a hyperplane, regardless of the activation function. This means that the perceptron is only a binary linear classifier and therefore can only separate inputs that are linearly separable, as shown in Fig. 1a. This poses a problem, even for simple cases where humans can easily separate the classes, as shown in Fig. $1 \mathrm{~b}$.

\subsubsection{Neural networks}

A neural network [10] is a system that aims to solve the perceptron's linearity problem by building layers of neurons and linking them together. A layer is a group of neurons that receive the same input and transmit their

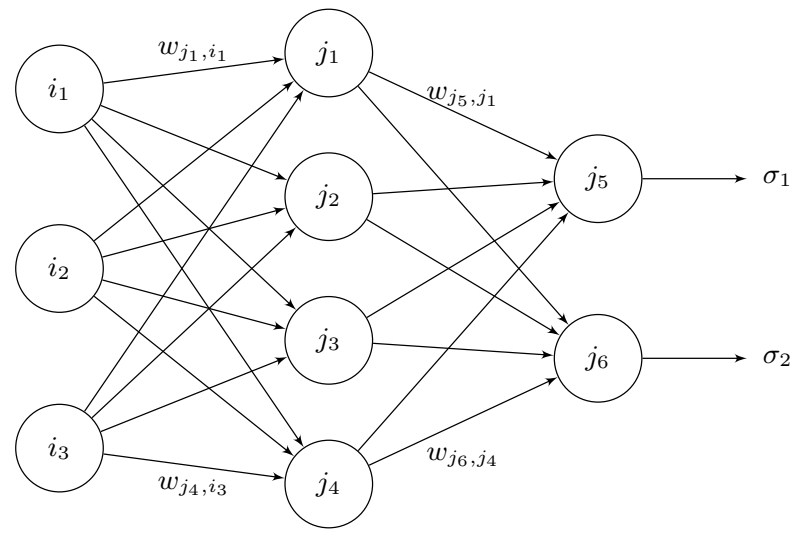

Input Layer Hidden Layer Output Layer

Fig. 2 Classic artificial neural network architecture. The input for each neuron $j$ is given by the weighted sum of all the neurons in the previous layer

output forward. A neural network is made of an input layer, one or more hidden layers and an output layer. Apart from the input layer, every neuron uses an activation function to determine its output, as was the case with the perceptron model. In its classic form, every neuron in a layer receives signals from every other neuron in the previous layer, calculates its output and transmits it to the next layer, until the output layer is reached and the network's output is computed. This process is illustrated in Fig. 2.

Formally, the input of each neuron $j$ in the first hidden layer is given by the sum of the output of every neuron $i$ in the input layer, $\sigma_{i}$, multiplied by the weight associated with input $i$ of neuron $j, w_{j, i}$ :

$n e t_{j}=\sum_{i=1}^{n} w_{j, i} \cdot x_{i}$.

As in the perceptron, the output of node $j$ is then calculated with an activation function. However, unlike in the perceptron model, this output is then fed again to some other neurons $k$ in the next layer as input, again multiplied by the corresponding weight, $w_{k, j}$. The activation function is yet again applied to this weighted sum, yielding the output for the second hidden layer:

$\sigma_{k}=f\left(\right.$ net $\left._{k}\right)=f\left(\sum_{j=1}^{n} w_{k, j} \cdot f\left(n e t_{j}\right)\right)$.

If the activation function $f$ is linear, the neural network will only be able to classify linearly separable inputs. However, with a non-linear activation function, the resulting decision boundary becomes the combination of different non-linear 
function regions, which allows for classification of linearly inseparable data.

\subsubsection{Learning in neural networks}

Learning in artificial neural networks consists of finding the right weights that drive the network to classify the input correctly. This task, however, is unfeasible to do by hand, even for a relatively small network, much less for a large deep neural network.

Stochastic gradient descent [15] is the method that is generally used to optimize the weights of a neural network. In [31], Rumelhart describes the generalized delta rule. The system proposed uses the input vector to produce an output vector $\sigma$, as described in the previous section, and then compares it to the desired output vector. If there is no difference whatsoever, there is no need to update anything in the network. Otherwise, the weights are slowly changed to minimize the difference between the two outputs. For each neuron $j$ in the network, its weight for input $i, w_{j, i}$ is updated using:

$\Delta w_{j, i}=\eta \delta_{j} \sigma_{i}+\alpha \Delta w_{j, i}$

$w_{j, i}=w_{j, i}+\Delta w_{j, i}$

where $\eta$ is the learning rate, $\sigma_{i}$ is the output of neuron $i$ and $\alpha$ a factor applied to the previous update. $\alpha \Delta w_{j, i}$ is called the momentum, and its purpose is to keep the updates in the same direction, minimizing the chance of being influenced by outliers. $\delta_{j}$ is given by:

$\delta_{j}=-\frac{\partial E}{\partial n e t_{j}}$.

$E$ is the error function that measures how much the network output deviated from the expected output. In the original proposal, the error of an output node $i$ was, as in $\operatorname{TD}(\lambda)$, the mean squared error:

$E=\frac{1}{2}\left(t-\sigma_{i}\right)^{2}$,

where $t$ is the actual target output.

We have to keep in mind that $\delta_{j}$ is calculated using the derivative of the error function relative to the net input of node $j$. However, the error function is relative to the output of the network. This means that, unless $j$ is an output node, we have to apply the chain rule to obtain the derivative. This process is called backpropagation, and its main objective is to propagate the error calculated in the output layer onto the hidden layers.

This is the main functionality of a neural network when used in supervised learning. In reinforcement learning, there is no target output, there is only a feedback reward returned from the environment in response to the action an agent takes. Nevertheless, given a set of input features from the environment, we can still train a neural network to predict the reward the agent will receive, if it takes a specified action, and then adjust the weights using the difference between the expected reward outputted by the network and the actual reward given by the environment. An action can be represented in the network by one output neuron, meaning that if the agent has $n$ actions the network would have $n$ output neurons. The update is performed the same way as described before.

Neural networks, as most methods in machine learning, are prone to overfitting. An overfit network learns the training data too well, including noise. This leads to a very good accuracy in the training phase, but a low score when classifying unseen cases. There are changes, called regularization techniques, that can be done to the network's architecture to avoid overfitting. Some common ones are the $L 2$ and the L1 weight decay [24] and the dropout method [39].

Furthermore, neural network layers may receive and output values that are not on the same scale. This means that in some cases an output from a neuron may be much larger than the others from the same layer, effectively overshadowing them on the weighted sum. Normalization techniques transform the data to counteract differences in scale. A very common method is batch normalization [13].

\subsubsection{Convolutional and residual neural networks}

Although convolutional neural networks (CNNs) are not a new concept [20], only recently have they gained more traction in the machine learning community [18], in particular due to the increase in GPU computing power, which allows for faster training of models like deep neural networks. Image recognition is where it has been used most.

CNNs are typically composed of convolutional, pooling, ReLU and fully connected layers, as shown in Fig. 3. For simplicity, let us assume grayscale images of size $W$ as input.

Convolutional layers transform an input by applying a filter. A filter is a matrix of size $F \times F$ where $F<<W$, and its purpose is to find features in the image, for instance, edges. The image is scanned with the filter, meaning that the dot product is applied to every group of $F \times F$ pixels in $W$ (assuming stride equal to 1 ), yielding a value (or an output) for each group. These values form an activation map which is typically smaller than the input. The scanning allows the neural network to learn to identify features regardless of the position in the input image, which is a mandatory requirement for image recognition. It is also possible to use multiple filters in the same layer, albeit one at a time, in order to identify multiple features. 


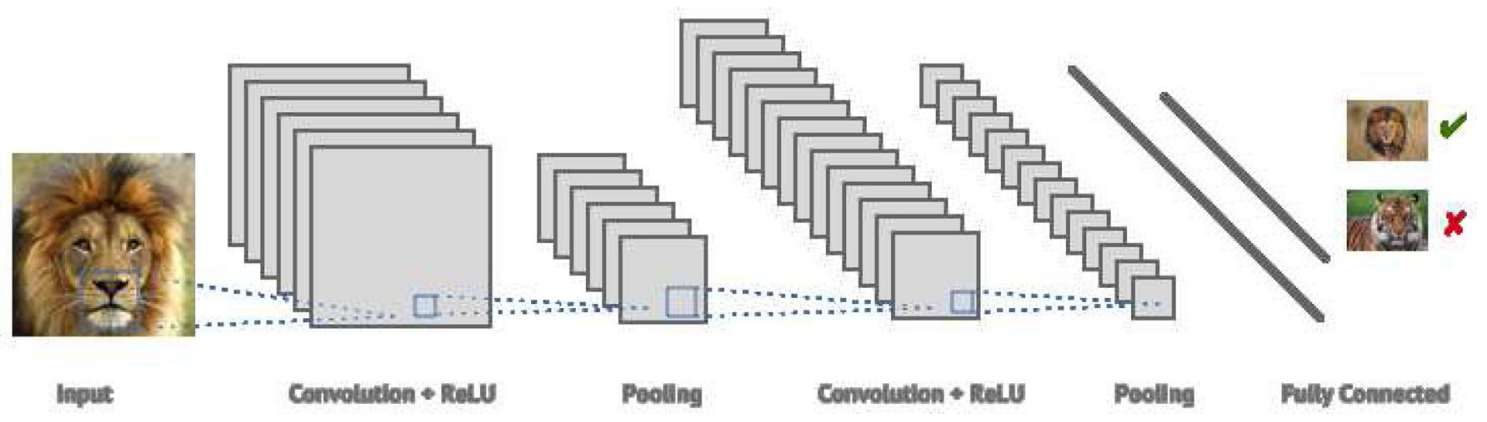

Fig. 3 Typical convolutional neural network architecture. The square on the image of the lion is the filter with size $F \times F$
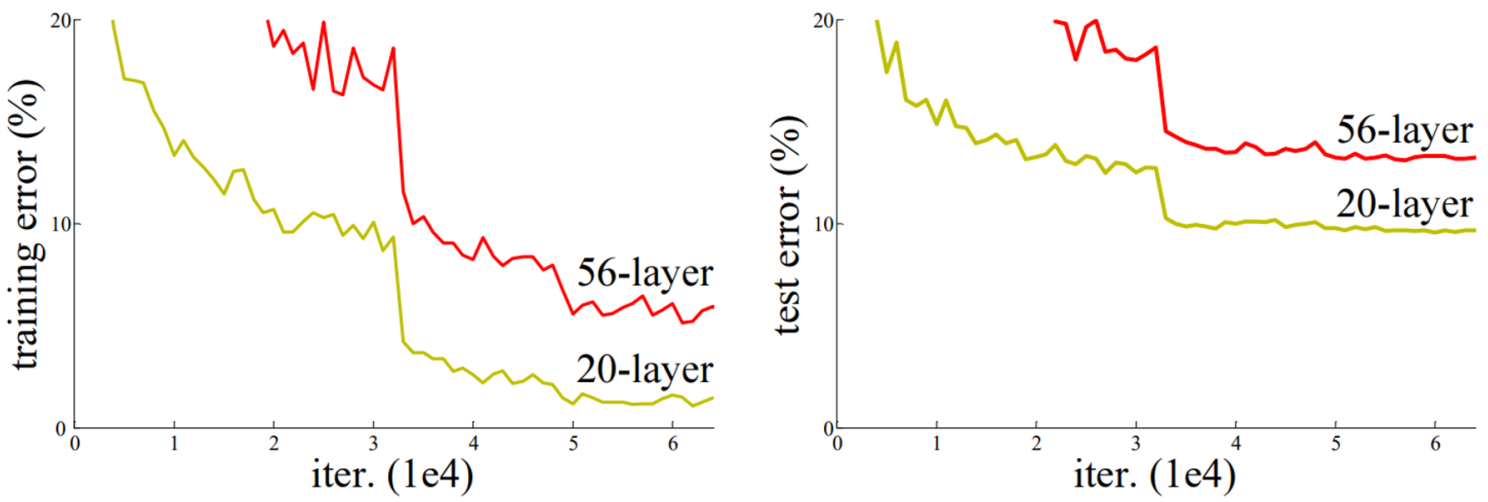

Fig. 4 Training error (left) and test error (right) on CIFAR-10 with 20-layer and 56-layer "plain" networks (extracted from [11]). Results show that, contrary to expectations, deeper networks do not necessarily have better performance

Since convolutional layers only apply a linear operation to the input, in order to separate non-linear data, a nonlinear layer must be added. The ReLu layers apply the rectifier linear unit function to the activation maps. For each value $x$ in the activation map,

$f(x)=\max (x, 0)$,

which effectively just replaces every negative value with 0 .

Pooling layers reduce the dimensionality of the input. The main idea is that the relative position of the features is much more important than the absolute position. The most common method of pooling is called max pooling [32], which divides the input into rectangles and outputs the maximum value. Pooling also helps speed by reducing the spatial size of the representation and, therefore, the amount of computations needed to train and use the network. Moreover, since there are effectively less parameters to adjust, it becomes harder for the network to overfit.

Theoretically, a neural network with just a single layer is enough to represent any function. However, its size could be too large for practical uses and it would be prone to overfitting. Another way of achieving better results is to create deeper neural networks with multiple hidden layers. Even though this works to some extent, the performance of deeper networks actually lowers as the number of layers increases (see Fig. 4). The main reason for this is the vanishing gradient problem [3]. During backpropagation, the consecutive multiplications the gradient endures may make the gradient very close to 0 .

Residual neural networks [11] are a special type of CNN that address the vanishing gradient problem by introducing skip connections to jump over some layers. In a typical neural network, a hidden layer $I_{k}$ only receives the output of the previous layer $I_{k-1}$ and its output serves as input to the next layer $I_{k+1}$ (see Fig. $5 \mathrm{a}$ ). We train the network in hope that the layers fit a desired mapping $H(x)$. A skip connection lets the output from $I_{k-1}$ also serve as input to the activation function of a layer ahead of $I_{k}, I_{k+n}$, with $n>0$, by adding it to the weighted output of layer $I_{K+n-1}$ (see Fig. $5 b$ ).

With this architecture, if the identity $x$ is already optimal, meaning that there are no more features left to extract, the weights can be set to 0 and no changes are made. However, if $x$ is only close to optimal, we train the network in hope that the skipped layers learn a residual mapping $F$ and fit any small fluctuation in features that $x$ might need to become closer to optimal and subsequently fit a desired mapping $H$. Further studies [12] suggest that for even deeper neural networks, the best results are achieved when 


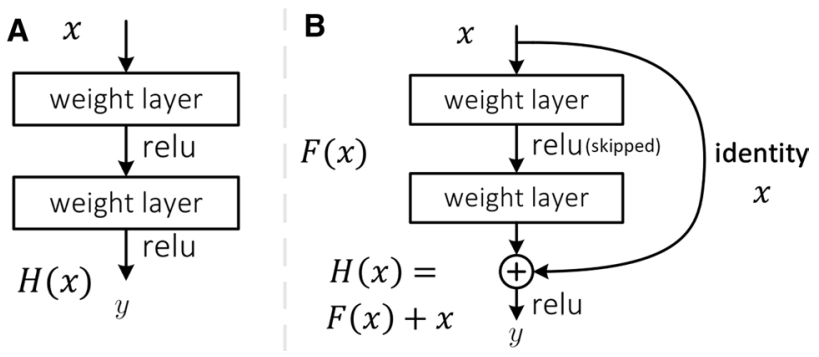

Fig. 5 Two layers in a plain network (a) and in a residual network (b) (adapted from [11]). In a, we want the layers to fit a desired mapping $H$, while in $\mathbf{b}$ we want them to fit a residual mapping $F$ that, together with the input $x$, fit $H$

the identity not only skips the weighting phase of the layer it is being plugged into, but also the activation function.

The backpropagation process is similar to the one previously described:

$$
\begin{aligned}
\frac{\delta E}{\delta x} & =\frac{\delta E}{\delta y} \frac{\delta y}{\delta x} \\
& =\frac{\delta E}{\delta y} \cdot\left(1+F^{\prime}(x)\right) \\
& =\frac{\delta E}{\delta y}+\frac{\delta E}{\delta y} \cdot F^{\prime}(x)
\end{aligned}
$$

As we can see, the derivative is now maintained in the first addend, thus minimizing the vanishing gradient descent problem.

\section{Related work}

\subsection{TD-gammon}

One of the first and most successful practical implementations of temporal difference learning was TD-Gammon [44], which explored the viability of training a neural network with one hidden layer using $\operatorname{TD}(\lambda)$.

This system achieved a strong level of play, approximately equal to Tesauro's previous work, NeuroGammon [43], a supervised learning approach consisting of seven neural networks, trained with examples from human games. TD-Gammon was then combined with NeuroGammon's hand-designed features, achieving even better performance. However, despite its excellent performance, TD-Gammon was never able to entirely beat the top-rated backgammon players at the time, perhaps due to the lack of computational power.

In practice, the neural network may be viewed as a nonlinear value function approximator $f$, like the one described in Sect. 2.3, in which the weights are the parameters.
The neural network received as input the raw features of the board, i.e., the amount of black or white checkers at each position, and produced an output consisting of a four component vector, corresponding to the possible outcomes of the game: White wins, Black wins, White wins with gammon or Black wins with gammon.

The neural network was used to select moves for both sides, training by playing against itself, starting from completely random play. At each time step, the dice were rolled and the possible actions and the respective board configurations were calculated. All the resulting board configurations were then fed to the network and classified. The action taken by the player was whichever yielded the best expected result for the current player.

After each time step, the weights are updated using the $\operatorname{TD}(\lambda)$ algorithm, using gradient descent as previously described:

$w_{t+1}=w_{t}+\alpha \nabla_{w} E$.

Starting from random play, TD-Gammon was able to learn the basics and some advanced tactics of backgammon. It discovered novel strategies which changed the way humans play and even top players modified their positional thinking and opening strategies based on the moves recommended by the system.

\subsection{Deep Q-learning in Atari games}

Shortly after being acquired by Google, DeepMind started working on deep Q-learning (DQN) [22]. This novel algorithm improves on Q-learning by using a neural network to approximate the Q-values of an action, given a particular state, similar to TD-Gammon. This allows the agent to learn without having to memorize all the possible state-action pairs and their respective $\mathrm{Q}$-values.

The algorithm was used to play a range of Atari 2600 games, using an emulator extended with a reinforcement learning framework created by [2] that allows the system to play at a much higher pace than real time, effectively speeding up the training phase.

The system receives raw frames from the emulator. However, each frame requires an excessive amount of computation, so they are firstly down-sampled, grayscaled and cropped to an $84 \times 84$ image that captures the playing area in black and white. The Atari games have a dynamic environment, which means that, unlike in backgammon or other board games, the environment changes, even if the agent does not do anything. For instance, in Pong, even if the player does not move the paddle, the ball keeps moving, and its movement is of the utmost importance. If a single frame is used, the agent cannot see the dynamic elements of the game, including its own movement. To solve 
this, instead of just one frame, a stack of four preprocessed frames is used as input for the neural network.

The output of the network consists of a layer with an output unit for each possible action. The outputs are the predicted Q-values for the corresponding action in the particular input state.

After feeding an input state $s_{t}$ to the network, the agent then selects and executes an action $a_{t}$ following the $\epsilon$ -greedy heuristic. The reward $r_{t}$ received and the new state $s_{t+1}$ are then observed and stored together with $s_{t}$ and $a_{t}$ in what is called a replay memory, that stores the last $N$ transitions that occurred, where $(N=1,000,000)$ for [22]. The transitions are then used for a technique known as experience replay [21]. At every time step, a batch of 32 randomly selected transitions is selected and an update using gradient descent is performed with an error function very similar to the one described in Background. The target $y_{j}$ used in the gradient is given by:

$y_{j}=\left\{\begin{array}{ll}r_{j} & \text { if } s_{t+1} \text { is terminal } \\ r_{j}+\gamma \max _{a^{\prime}} \hat{Q}\left(s_{t+1}, a^{\prime}\right) & \text { otherwise }\end{array}\right.$,

where $\hat{Q}$ is the actual function that calculates the max $Q$-value for the next-state. This is an exact copy of $Q$ made every $C$ time steps, which means that the targets are actually given by a value function that is "behind" on the learning process. Nevertheless, this reduces variations due to changes in the policy and yields a more stable learning. Experience replay also helps in data efficiency, by allowing one sample to be used multiple times. Since each time step is very much related to the previous one, the updates become correlated. However, by selecting randomly from a large pool of transitions, this correlation is broken.

The algorithm for deep Q-learning is described in Algorithm 3:

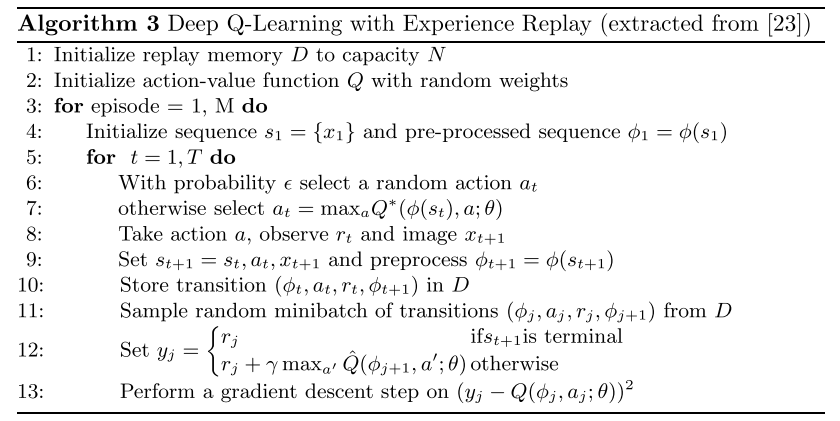

The network itself is made up by the input layer described above followed by three pairs of layers, each made up by one convolutional layer and one ReLU layer. The convolutional layers were fundamentally equal to the ones in Sect. 2.5 and in [18]. The last hidden layer is fully connected, and the output is composed of a neuron for each valid action, which varied from game to game.
The results obtained show that deep Q-learning surpassed professional human games testers in the majority of games. Moreover, further testing shows that experience replay revealed to be one of the most important factors in achieving such a great performance in some games, and using $\hat{Q}$ as a target was determining in others. Regardless, the combination of both these methods yielded the very best results.

\subsection{AlphaGo}

Traditional Go or chess engines use effective search to find the best move for each position. One common approach is to reduce the depth of the search by predicting the outcome of the game using an approximate value function, once a certain depth is reached. By only using this method, former chess world champion Garry Kasparov was beaten by Deep Blue [6]; however, due to the complexity of Go, this method is unfeasible. The breadth of the search can also be reduced using the Monte Carlo tree search (MCTS) algorithm, which essentially uses a policy to sample the following actions and calculate through experience the expected outcome for taking a specific action. Regardless, with both these methods, only amateur-level play was achieved in Go [4].

Following the success of deep Q-Learning in Atari games, DeepMind decided to tackle the board game of Go [35], where, unlike checkers and chess, humans were still stronger than machines.

The system was composed of three artificial neural networks: a large, deep policy network $p_{\sigma}$; a smaller, faster policy network $p_{\pi}$; and a value network $V_{\theta}$. The policy network $p_{\sigma}$ was composed of the input layer, 12 pairs of convolutional and ReLU layers and an output layer with as many neurons as actions, as seen in DQN. The main difference between this network and the previous is that this one uses action probabilities instead of $\mathrm{Q}$-values. The value function had a similar structure to $p_{\sigma}$, with the exception of the output layer, that had just one neuron that predicted the probability of winning the game.

Firstly, both policy networks were trained via supervised learning using expert human moves from the KGS Go Server. This network was able to predict the next best move with an accuracy of about $56 \%$ using just raw board position and move history as inputs.

Secondly, $p_{\sigma}$ was improved via reinforcement learning, by playing against random previous versions of itself. The improved network was called $p_{\rho}$. Self-play helps the system win games, rather than just predicting the next move. Randomly selecting opponents helps to stabilize training and prevent overfitting. The rewards used were 

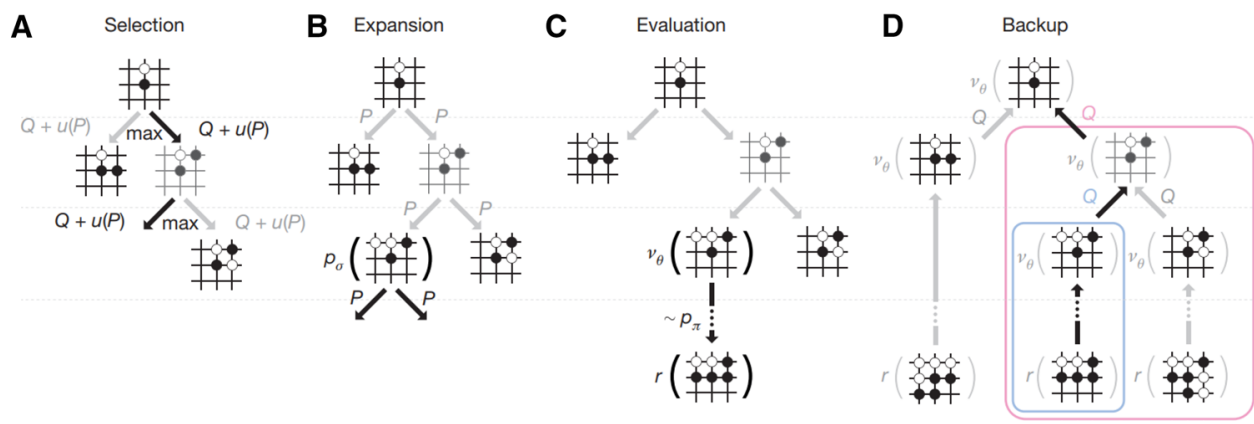

Fig. 6 Monte Carlo tree search in AlphaGo (extracted from [35]). a Each simulation traverses the tree by selecting the edge with maximum action value $Q$, plus a bonus $u(P)$ that depends on a stored prior probability $P$ for that edge. $\mathbf{b}$ The leaf node is expanded, and the new node is processed once by $p_{\rho}$. The output probabilities are

simply +1 if the game was won, -1 if it was lost and 0 every non-terminal time step.

Finally, the value function was trained to predict the winner in a new set of games where the policy network played against itself. Each game had a sample board position extracted at a random time step, which was then used to train $V_{\theta}$. The value function achieved a very good accuracy, coming close to Monte Carlo rollouts using $p_{\rho}$.

Just by itself, the policy network $p_{\rho}$ was able to beat Pachi [1], an advanced amateur-level engine based on MCTS, $85 \%$ of the times. However, AlphaGo's team decided to use the networks to guide a MCTS algorithm, as described in Fig. 6 . If a move is legal, it is represented in the tree by an edge which stores an action value $Q(s, a)$, a visit count $N(s, a)$ and the prior probability from the network $P(s, a)$. During the Monte Carlo simulations, the action for time step $t, a_{t}$ is selected as follows:

$a_{t}=\underset{a}{\arg \max }\left(Q\left(s_{t}, a\right)+u\left(s_{t}, a\right)\right)$,

where $u$ is a modified version of the PUCT algorithm [29]:

$u(s, a)=C \cdot P(s, a) \frac{\sqrt{\sum_{b} N(s, b)}}{N(s, a)}$

where $C$ is a constant that determines the level of exploration. The value network is used to evaluate the leaf node selected by the algorithm, I. A rollout is then performed starting from / and following the fast policy $p_{\pi}$ until an end state is reached. The statistics for every edge from node $s$ to node $I$ are then updated using the evaluation and the result from the rollout. After a predefined amount of time has passed, the MCTS chooses an action to take based on the number of times each action in node $s$ was selected.

This system became the most powerful entity in Go when in 2016 beat the world champion Lee Sedol 4 to 1. then stored as prior probabilities $P$ for each action. $c$ The leaf node is evaluated using the value network and by running a rollout to the end of the game with the fast rollout policy $p_{\pi}$. In the end, the reward is given. d Action values $Q$ are updated to track the mean value of all evaluations $r$ and $V_{\theta}$ in the subtree below that action

\subsection{Asynchronous advantage actor critic}

Asynchronous Advantage Actor Critic (A3C) [23] is an actorcritic algorithm that was the best of four asynchronous variants of standard RL algorithms proposed by the DeepMind team.

$\mathrm{A} 3 \mathrm{C}$ also has a policy $\pi_{\theta}$ and a value function $V_{\theta v}$, which are updated using the forward view of $n$-step Q-learning. Both have different parameters $\left(\theta\right.$ for the policy and $\theta_{v}$ for the value function) and are updated after every $t_{\max }$ actions or when a terminal state is reached. Due to the fact that we are dealing with probabilities rather than values, the updates for the parameters for policy $\pi$ are given by gradient ascent using

$\nabla_{\theta} \log \pi\left(a_{t} \mid s_{t} ; \theta\right) A\left(s_{t}, a_{t} ; \theta, \theta_{v}\right)$,

where $A$ is the advantage function that represents how much better the agent will get by taking action $a_{t}$, when compared to the average value for that state. The advantage function is computed in a similar fashion as in TD Learning, as follows:

$A\left(s_{t}, a_{t}\right)=\sum_{i=0}^{k-1} \gamma^{i} r_{t+i}+\gamma^{k} V\left(s_{t+k} ; \theta_{v}\right)-V\left(s_{t} ; \theta_{v}\right)$

The actor-critic part comes from using the learned value function estimate (critic) to scale the policy (actor) gradient. This leads to a much lower variance in the estimate of the policy gradient. Furthermore, the method described combines both estimates in the same neural network by splitting it in two output layers and by sharing most hidden layers. The idea behind the division is to use the first part of the network as a feature extractor and have both heads output two different things, while sharing the same parameters in the first layers. This system was first proposed by [7]. 
Finally, the asynchronous part comes from running multiple agents on several instances of the task's environment. Each agent has its own independent neural network and asynchronously updates a global neural network after a pre-determined amount of epochs. This way, the agents experience different examples, leading to a much more diverse training, which is especially crucial in this case since the steps in $n$-step algorithms have a very high correlation.

This algorithm surpassed DeepMind's own deep Q-learning in the Atari games requiring only half the time DQN originally had and while training in a multi-core CPU rather than a GPU. Moreover, this method also succeeded in a variety of other tasks, such as navigating mazes, as described in [23].

\subsection{AlphaGo Zero and Alpha Zero}

Even though AlphaGo was able to defeat the Go world champion, it still relied on human expert examples to train via supervised learning. These data in other real-world cases are often not available for multiple reasons, and even when it is, it often sets a ceiling on the performance of the system. So DeepMind decided to create another version of AlphaGo called AlphaGo Zero (AGZ) [38], which trained by playing against itself, without any supervision and by only looking at the stones in the board.

The first major difference in the architecture of AlphaGo Zero is the single neural network it uses, instead of the three AlphaGo used. AGZ's network receives not only the current board position, but also the board from the last 7 time steps, due to the fact that in Go, it is illegal to place a stone that results in a repetition of the board. Additionally, the network also knows which player's turn it is through a feature plane all set to either 1 or 0 .

The input is processed by a convolutional block, made up by a convolutional layer with 256 filters of size $3 \times 3$ with stride 1, a batch normalization [13] layer and a ReLU layer. The convolutional block is then followed by 39 residual blocks. Each residual block is composed of two convolutional blocks like the one described above, with the exception that the second convolutional block has a skip connection between the normalization and ReLU layers.

The network then diverges in two "heads" as in A3C. The policy head is composed of a convolutional layer with two filters of size $1 \times 1$ with stride 1 , a batch normalization layer, a ReLU layer and the output layer, which contains a neuron for every intersection on the board and one more for the pass move. The value head is composed of a convolutional layer with one filter of size $1 \times 1$ with stride 1 , a batch normalization layer, a ReLU layer, a fully connected linear layer of size 256 , followed by another ReLU layer, another fully connected linear layer and a neuron that applies the tanh function, outputting a scalar between -1 and 1 .

The system then uses the information from the policy to bias a MCTS starting from the current board position in the root node, just like AlphaGo. However, unlike AlphaGo, there are no rollouts when a leaf node $I$ is reached. Instead, another forward pass through the neural network is made with the board position from node $I$. The resulting probabilities from the policy head are then used to expand $l$, and the evaluation from the value network is used to update the action value $Q$ of every node selected from the root node to $I$. For every edge $(s, a)$ selected, the update is as follows:

$Q(s, a)=\frac{1}{N(s, a)} \sum_{s^{\prime} \mid s, a \rightarrow s^{\prime}} V\left(s^{\prime}\right)$,

where $s^{\prime} \mid s, a \rightarrow s^{\prime}$ represents every node the MCTS selected from the moment it chose action $a$ in state $s$ until it reached $s^{\prime}$.

After a predefined amount of time, the algorithm chooses whichever action had the best $Q$-value in the root node. The network parameters are then updated using the search probabilities $\pi$ that the MCTS counts yield. However, AlphaGo Zero keeps a main player with its own neural network that is used to generate data from self-play. Other secondary networks sample these data in mini batches and update themselves. From time to time, the neural networks are put up against the primary network. If a secondary network can beat the primary network by a margin of over $55 \%$, it becomes the primary network and is subsequently used to generate more data.

A more general system called Alpha Zero (AZ) $[36,37]$ was also created. This system can not only play Go, but also chess and shogi. The neural network architecture, despite being better suited for Go, is the same as in AGZ, with the exception of the input and output layers, which depend on the game being played. Nevertheless, Alpha Zero differs from AGZ in a few key aspects. Most notably, while AGZ took advantage of the symmetry Go had and could augment its dataset using these symmetries, chess and shogi are asymmetrical and AZ does not assume symmetry. AGZ only updated its main player when a an update could defeat its current one by a margin of $55 \%$. In contrast, Alpha Zero uses a more simple approach and only has one network that is updated continuously. 
Alpha Zero achieved superhuman performance in all three games, beating all previous engines.

One of the most promising attempts at replicating the success of Alpha Zero is Leela Zero, ${ }^{1}$ and its chess-only counterpart Leela Chess Zero ${ }^{2}$ (LCO). It is trained in a distributed way across anyone who wishes to help, rather than on DeepMind's supercomputer. So far, its strength is still surpassed by the claimed performance of Alpha Zero, but it is showing promising results, especially in chess, where it has become one of the strongest engines.

\subsection{TRPO and PPO}

Trust region policy optimization (TRPO) [33] is a policy gradient method that targets the high variance of other algorithms such as the REINFORCE.

The objective is still to maximize the expected reward $J$. We can estimate how much better an updated policy $\pi^{\prime}$ will do when compared to an old policy $\pi$ by replacing the estimate $Q^{\pi}$ in the original formula by an estimate of the advantage function $A^{\pi}$ and using the expected reward from $\pi$. It has been proven by [14] that by representing the expected reward of the new policy in terms of the advantage of another policy as follows, we are guaranteed that an increase in performance is possible.

$J\left(\theta^{\prime}\right)=J(\theta)+\sum_{s} P(s) \sum_{a} \pi^{\prime}(a \mid s) A^{\pi}(s, a)$.

The expected reward for the old policy $J(\theta)$ can be treated as a constant, which means that one can deduce the following:

$$
\begin{aligned}
\underset{\theta^{\prime}}{\arg \max J\left(\theta^{\prime}\right)} & =\underset{\theta^{\prime}}{\arg \max } J\left(\theta^{\prime}\right)-J(\theta) \\
& =\underset{\theta^{\prime}}{\arg \max } \sum_{s} P(s) \sum_{a} \pi^{\prime}(a \mid s) A^{\pi}(s, a) \\
& =\underset{\theta^{\prime}}{\arg \max } \mathbb{E}_{s \sim P(s), a \sim \pi^{\prime}}\left[A^{\pi}(s, a)\right],
\end{aligned}
$$

This implies taking samples from $\pi^{\prime}$ for every state $s$ to find out the distribution for the actions. However, this is very inefficient, since every time the network changes, new samples have to be taken. To counteract this, we can reuse samples taken from the older policy $\pi$ by applying importance sampling [9] to the expected value equation, as in Eq. (43):

$$
\mathbb{E}_{s \sim P(s), a \sim \pi^{\prime}}\left[A^{\pi}(s, a)\right]=\mathbb{E}_{s \sim P(s), a \sim \pi}\left[\frac{\pi^{\prime}(a, s)}{\pi(a, s)} A^{\pi}(s, a)\right]=L_{\theta_{o l d}}^{\prime s}\left(\theta^{\prime}\right),
$$

thus obtaining a new loss function, $L_{\theta_{\text {old }}}^{I S}\left(\theta^{\prime}\right)$ that approximates the actual performance difference between $\pi^{\prime}$ and $\pi$, as shown by [14, 33]. Moreover, both the expected value and the advantage function can be replaced by an estimator at a generic time step $t$, respectively, $\hat{\mathbb{E}}_{t}$ and $\hat{A}_{t}$. By taking an approach similar to Eqs. (19)-(21), the following gradient is obtained:

$$
\begin{aligned}
\nabla_{\theta^{\prime}} L_{\theta_{\text {old }}}^{\text {LS }}\left(\theta^{\prime}\right) & =\hat{\mathbb{E}}_{t}\left[\frac{\nabla_{\theta^{\prime}} \pi^{\prime}\left(a_{t}, s_{t}\right)}{\pi\left(a_{t}, s_{t}\right)} \hat{A}_{t}\right] \\
& =\hat{\mathbb{E}}_{t}\left[\frac{\pi^{\prime}\left(a_{t}, s_{t}\right)}{\pi\left(a_{t}, s_{t}\right)} \nabla_{\theta^{\prime}} \log \left(\pi^{\prime}\left(a_{t}, s_{t}\right)\right) \hat{A}_{t}\right]
\end{aligned}
$$

.TRPO aims to maximize $L_{\theta_{\text {old }}}^{\text {IS }}\left(\theta^{\prime}\right)$ in order to improve the policy, but without straying too far from the previous policy. This is done via a constraint that employs the Kullback-Leibler (KL) divergence [19], a measure of how much two probabilities differ from one another:

$$
\begin{array}{cl}
\underset{\theta}{\arg \max } & L_{\theta_{\text {old }}}^{\text {IS }}\left(\theta^{\prime}\right) \\
\text { subject to } & \hat{\mathbb{E}}_{t}\left[\operatorname{KL}\left[\pi, \pi^{\prime}\right]\right] \leq \delta
\end{array}
$$

where $\delta$ is a hyperparameter that represents the distance.

Proximal policy optimization [34] is an improvement over TRPO as it simplifies most computations required by the constraint. PPO modifies TRPO's loss function to include a very simple penalty for having an update that is too big. Let the ratio between the new policy and the old one be $\varphi_{t}(\theta), L^{\mathrm{CLIP}}$ denotes the new objective function:

$L^{\mathrm{CLIP}}(\theta)=\hat{\mathbb{E}}\left[\min \left(\varphi_{t}(\theta) \hat{A}_{t}, \operatorname{clip}\left(\varphi_{t}(\theta), 1-\epsilon, 1+\epsilon\right) \hat{A}_{t}\right)\right]$.

As illustrated in Fig. 7, when $A>0$, the objective function will not allow the new policy to diverge more than $1+\epsilon$ from the old one. The same happens, albeit in reverse, when $A<0$.

This system has been applied to a multitude of environments, most recently, a multi-agent Hide and Seek [5] and Dota $2 .^{3}$ The results are very impressive and promising. Of course, a quick look at the hardware needed to achieve such results reveals the immense computational power required to back them up, which means there is still room for improvement. Regardless, we can already see the potential these algorithms have to improve and even revolutionize other fields besides games. For instance, while learning to play hide and seek, the agents discovered some exploits that could give them a significant advantage over the adversary. These exploits were unknown even to

\footnotetext{
3 https://blog.openai.com/openai-five/.
}

\footnotetext{
1 https://zero.sjeng.org/.

2 https://lczero.org/.
} 


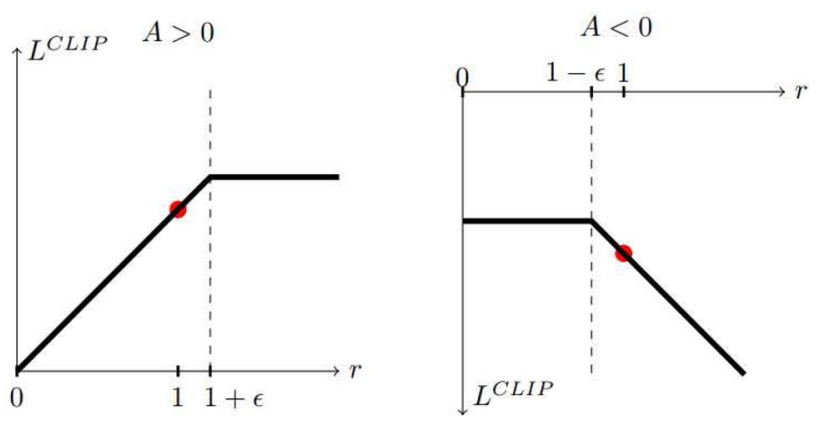

Fig. 7 Plots showing a single time step of $L^{\text {CLIP }}$ as a function of the probability ratio $\varphi$, for positive advantages (left) and negative advantages (right). The red circle on each plot shows the starting point for the optimization, i.e., $r=1$ (extracted from [34])

the creator of the environment. Should these occurrences happen in other fields, it could help researches to identify previously ignored or unknown signs.

\section{Conclusions}

Machine learning is a concept that has been around for many decades, yet only now can we start to explore some of the principles proposed, test their limits, adapt and improve them.

While supervised learning methods work very well in cases where data need to be analyzed, in many real-world cases such data are unavailable or unfeasible. Reinforcement learning algorithms, on the other hand, are best suited for problems where an analytical solution is not well defined, such as most cases in the field of robotics and games.

In the last few years, we have witnessed an increase in the application of reinforcement learning techniques to games, such as AlphaGo and AlphaZero. Although these systems achieved very good results, there is still room for improvement, especially if we consider continuous and dynamic environments such as StarCraft, where only now progress is starting to unfold [46]. This trend may lead to unexpected and important achievements in other fields, like medicine and robotics.

\subsection{Current limitations}

The continuous increase in computational power allowed the community to overcome many barriers that were present in the early days of ML. Many more techniques and architectures, such as convolutional neural networks have been made possible not only due to this increase, but also because of the integration of hardware more suited for ML work than general-purpose CPUs, namely GPUs. Even hardware specialized for machine learning has been created, like Google's TPU. However, the biggest limitation we face in this area is still hardware-related. Many successes in this field, and perhaps especially when dealing with reinforcement learning, are only achievable with a substantial amount of resources, not available to many companies, let alone individual developers.

This induces a false sense of accomplishment within the community where researchers believe they can reach the results published by bigger companies, when in reality, even armed with the knowledge to do so, they may suffer from a lack of resources. Of course, this is the way to make progress in science, and larger companies will always be at the front-end of discovery. Nevertheless, there is a lack of transparency and reproducibility in many of the works published. For instance, the AlphaGo and AlphaZero papers $[35,37]$ have a good transparency regarding the methods employed and the networks used. However, they also lack reproducibility by not providing any data, code or even hyperparameters used during training. This leads many groups to attempt and fail to fully reproduce the results. This is a widespread problem not exclusive to computer science, and there are even some organizations that are now raising awareness and trying to fight this issue.

\subsection{Future work}

In the future, we should shift our focus from trying to achieve more impressive results with more powerful hardware, to understanding exactly how humans learn through reinforcement learning and how good they are at it. The development of more data-efficient algorithms is crucial to avoid endless training times.

Moreover, bridging the gap between the simulation performed on a computer and the actual application in the real world is something necessary and which is not always done. Problems such as autonomous driving can potentially be solved or improved in the future with the help of RL algorithms, and it would be interesting to see and study them outside of the virtual realm. A simulationbased approach could help close this gap.

\subsection{Final remarks}

In this paper, we provided an overview of the classic machine learning techniques, such as artificial neural networks and temporal difference learning algorithms, as well as more modern ones, namely residual neural networks and more modern policy gradient techniques. Furthermore, we talked about the most important machine learning systems and algorithms applied to games, the most recent ones, AlphaZero and proximal policy optimization. 
With this overview, we hope to have improved the reader's understanding of the current state of reinforcement learning applied to games.

\section{Compliance with ethical standards}

Conflict of interest On behalf of all authors, the corresponding author states that there is no conflict of interest.

\section{References}

1. Baudiš P, Gailly J (2012) Pachi: state of the art open source go program. In: van den Herik HJ, Plaat A (eds) Advances in computer games. Springer, Berlin, pp 24-38

2. Bellemare MG, Naddaf $Y$, Veness J, Bowling M (2015) The arcade learning environment: an evaluation platform for general agents. In: Proceedings of the 24th international conference on artificial intelligence, IJCAI'15, AAAI Press, pp 4148-4152. http://dl.acm. org/citation.cfm?id=2832747.2832830

3. Bengio Y, Simard P, Frasconi P (1994) Learning long-term dependencies with gradient descent is difficult. IEEE Trans Neural Netw 5(2):157-166. https://doi.org/10.1109/72.279181

4. Bouzy B, Helmstetter B (2004) Monte-Carlo Go developments. Springer, Boston, pp 159-174. https://doi.org/10.1007/978-0387-35706-5_11

5. Baker B, Kanitscheider I, Markov T, Wu Y, Powell G, McGrew B, Mordatch I (2019) Emergent tool use from multi-agent autocurricula. arXiv e-prints arXiv: 1909.07528

6. Campbell M, Hoane A, hsiung Hsu F (2002) Deep blue. Artif Intell 134(1):57-83. https://doi.org/10.1016/S0004-3702(01)00129-1

7. Caruana R (1993) Multitask learning: a knowledge-based source of inductive bias. In: Proceedings of the tenth international conference on machine learning, Morgan Kaufmann, pp 41-48

8. Fauw JD, Ledsam JR, Romera-Paredes B, Nikolov $S$, Tomasev N, Blackwell S, Askham H, Glorot X, O'Donoghue B, Visentin D, van den Driessche $G$, Lakshminarayanan B, Meyer C, Mackinder F, Bouton S, Ayoub K, Chopra R, King D, Karthikesalingam A, Hughes CO, Raine R, Hughes J, Sim DA, Egan C, Tufail A, Montgomery $H$, Hassabis D, Rees G, Back T, Khaw PT, Suleyman M, Cornebise J, Keane PA, Ronneberger O (2018) Clinically applicable deep learning for diagnosis and referral in retinal disease. Nat Med 24(9):1342-1350. https://doi.org/10.1038/s4159 1-018-0107-6

9. Glynn PW, Iglehart DL (1989) Importance sampling for stochastic simulations. Manag Sci 35(11):1367-1392

10. Grossberg S (1988) Nonlinear neural networks: principles, mechanisms, and architectures. Neural Netw 1(1):17-61. https ://doi.org/10.1016/0893-6080(88)90021-4

11. He K, Zhang X, Ren S, Sun J (2016) Deep residual learning for image recognition. In: 2016 IEEE conference on computer vision and pattern recognition (CVPR), pp 770-778. https:// doi.org/10.1109/CVPR.2016.90

12. He K, Zhang X, Ren S, Sun J (2016) Identity mappings in deep residual networks. In: Computer vision-ECCV 2016, Springer International Publishing, pp 630-645. https://doi. org/10.1007/978-3-319-46493-0_38

13. Ioffe S, Szegedy C (2015) Batch normalization: accelerating deep network training by reducing internal covariate shift. In: Bach F, Blei D (eds) Proceedings of the 32nd international conference on machine learning. Proceedings of machine learning research, vol 37, PMLR, Lille, France, pp 448-456. http://proce edings.mlr.press/v37/ioffe15.html

14. Kakade S, Langford J (2002) Approximately optimal approximate reinforcement learning. In: Proceedings of the nineteenth international conference on machine learning, ICML '02, Morgan Kaufmann Publishers Inc., San Francisco, CA, USA, pp 267-274. http://dl.acm.org/citation.cfm?id=645531.656005

15. Kiefer J, Wolfowitz J (1952) Stochastic estimation of the maximum of a regression function. Ann Math Stat 23(3):462-466. https://doi.org/10.1214/aoms/1177729392

16. Knuth DE, Moore RW (1975) An analysis of alpha-beta pruning. Artif Intell 6(4):293-326. https://doi.org/10.1016/00043702(75) $90019-3$

17. Konda VR, Tsitsiklis JN (2003) OnActor-critic algorithms. SIAM J Control Optim 42(4):1143-1166

18. Krizhevsky A, Sutskever I, Hinton GE (2012) Imagenet classification with deep convolutional neural networks. In: Proceedings of the 25th international conference on neural information processing systems, vol 1, NIPS'12, Curran Associates Inc., USA, pp 1097-1105

19. Kullback S, Leibler RA (1951) On information and sufficiency. Ann Math Stat 22(1):79-86. https://doi.org/10.1214/aoms/11777 29694

20. Lecun Y, Bottou L, Bengio Y, Haffner P (1998) Gradientbased learning applied to document recognition. Proc IEEE 86(11):2278-2324. https://doi.org/10.1109/5.726791

21. Lin LJ (1992) Reinforcement learning for robots using neural networks. PhD thesis, Pittsburgh, PA, USA, uMI Order No. GAX93-22750

22. Mnih V, Kavukcuoglu $K$, Silver $D$, Rusu AA, Veness J, Bellemare MG, Graves A, Riedmiller M, Fidjeland AK, Ostrovski G, Petersen S, Beattie C, Sadik A, Antonoglou I, King H, Kumaran D, Wierstra D, Legg S, Hassabis D (2015) Human-level control through deep reinforcement learning. Nature 518(7540):529-533. https://doi. org/10.1038/nature14236

23. Mnih V, Puigdomènech Badia A, Mirza $M$, Graves A, Lillicrap TP, Harley T, Silver D, Kavukcuoglu K (2016) Asynchronous methods for deep reinforcement learning. arXiv e-prints arXiv:1602.01783

24. Ng AY (2004) Feature selection, I1 versus 12 regularization, and rotational invariance. In: Proceedings of the twenty-first international conference on machine learning, ICML'04, ACM, New York, NY, USA, $\mathrm{p} 78$

25. Pavlov IP (1941) Lectures on conditioned reflexes. In: Conditioned reflexes and psychiatry, vol II, International Publishers, New York

26. Raschka S (2014) Naive Bayes and text classification I: introduction and theory. arXiv e-prints arXiv: 1410.5329

27. Evans R, Jumper J, Kirkpatrick J, Sifre L, Green TFG, Qin C, Zidek A, Nelson A, Bridgland A, Penedones H, Petersen S, Simonyan K, Crossan S, Jones DT, Silver D, Kavukcuoglu K, Hassabis D, Senior AW (Dec 2018) De novo structure prediction with deep-learning based scoring

28. Rosenblatt F (1958) The perceptron: a probabilistic model for information storage and organization in the brain. Psychol Rev 65:386-408

29. Rosin CD (2011) Multi-armed bandits with episode context. Ann Math Artif Intell 61(3):203-230. https://doi.org/10.1007/s1047 2-011-9258-6

30. Ruder S (2016) An overview of gradient descent optimization algorithms. arXiv e-prints arXiv:1609.04747

31. Rumelhart DE, Hinton GE, Williams RJ (1986) Parallel distributed processing: explorations in the microstructure of cognition, Learning internal representations by error propagation, vol 1, MIT Press, Cambridge, MA, pp 318-362. http://dl.acm.org/citat ion.cfm?id=104279.104293 
32. Scherer D, Müller A, Behnke S (2010) Evaluation of pooling operations in convolutional architectures for object recognition. In: Artificial neural networks-ICANN 2010, Springer, Berlin, pp 92-101. https://doi.org/10.1007/978-3-642-15825-4_10

33. Schulman J, Levine S, Moritz P, Jordan M, Abbeel P (2015) Trust region policy optimization. In: Proceedings of the $32 \mathrm{Nd}$ international conference on international conference on machine learning, ICML'15, JMLR.org, vol 37, pp 1889-1897. http://dl.acm.org/ citation.cfm?id=3045118.3045319

34. Schulman J, Wolski F, Dhariwal P, Radford A, Klimov O (2017) Proximal policy optimization algorithms. arXiv e-prints arXiv :1707.06347

35. Silver D, Huang A, Maddison CJ, Guez A, Sifre L, van den Driessche G, Schrittwieser J, Antonoglou I, Panneershelvam V, Lanctot M, Dieleman S, Grewe D, Nham J, Kalchbrenner N, Sutskever I, Lillicrap T, Leach M, Kavukcuoglu K, Graepel T, Hassabis D (2016) Mastering the game of go with deep neural networks and tree search. Nature 529(7587):484-489. https://doi.org/10.1038/natur e16961

36. Silver D, Hubert T, Schrittwieser J, Antonoglou I, Lai M, Guez A, Lanctot M, Sifre L, Kumaran D, Graepel T, Lillicrap T, Simonyan K, Hassabis D (2017) Mastering Chess and Shogi by self-play with a general reinforcement learning algorithm. arXiv e-prints arXiv :1712.01815

37. Silver D, Hubert T, Schrittwieser J, Antonoglou I, Lai M, Guez A, Lanctot M, Sifre L, Kumaran D, Graepel T, Lillicrap T, Simonyan K, Hassabis D (2018) A general reinforcement learning algorithm that masters chess, shogi, and go through self-play. Science 362(6419):1140-1144. https://doi.org/10.1126/science.aar6404

38. Silver D, Schrittwieser J, Simonyan $K$, Antonoglou I, Huang A, Guez A, Hubert T, Baker L, Lai M, Bolton A, Chen Y, Lillicrap T, Hui F, Sifre L, van den Driessche G, Graepel T, Hassabis D (2017) Mastering the game of go without human knowledge. Nature 550(7676):354-359. https://doi.org/10.1038/nature24270

39. Srivastava N, Hinton G, Krizhevsky A, Sutskever I, Salakhutdinov $R$ (2014) Dropout: a simple way to prevent neural networks from overfitting. J Mach Learn Res 15:1929-1958

40. Sutton RS (1988) Learning to predict by the methods of temporal differences. Mach Learn 3(1):9-44. https://doi.org/10.1007/bf001 15009
41. Sutton RS, Barto AG (2018) Reinforcement learning: an introduction. MIT Press, New York

42. Sutton RS, McAllester D, Singh S, Mansour Y (1999) Policy gradient methods for reinforcement learning with function approximation. In: Proceedings of the 12th international conference on neural information processing systems, NIPS'99, MIT Press, Cambridge, MA, USA, pp 1057-1063. http://dl.acm.org/citation. cfm?id=3009657.3009806

43. Tesauro G (1990) Neurogammon: a neural-network backgammon program. In: 1990 IJCNN international joint conference on neural networks, vol 3, pp 33-39. https://doi.org/10.1109/IJCNN .1990 .137821

44. Tesauro G (1995) Temporal difference learning and td-gammon. Commun ACM 38(3):58-68. https://doi.org/10.1145/20333 0.203343

45. Tokic M (2010) Adaptive $\epsilon$-greedy exploration in reinforcement learning based on value differences. In: KI 2010: advances in artificial intelligence, Springer, Berlin, pp 203-210. https://doi. org/10.1007/978-3-642-16111-7_23

46. Vinyals O, Babuschkin I, Chung J, Mathieu M, Jaderberg M, Czarnecki WM, Dudzik A, Huang A, Georgiev P, Powell R, Ewalds T, Horgan D, Kroiss M, Danihelka I, Agapiou J, Oh J, Dalibard V, Choi D, Sifre L, Sulsky Y, Vezhnevets S, Molloy J, Cai T, Budden D, Paine T, Gulcehre C, Wang Z, Pfaff T, Pohlen T, Wu Y, Yogatama D, Cohen J, McKinney K, Smith O, Schaul T, Lillicrap T, Apps C, Kavukcuoglu K, Hassabis D, Silver D (2019) AlphaStar: mastering the real-time strategy game StarCraft II. https://deepmind.com/blog/alpha star-mastering-real-time-strategy-game-starcraft-ii/

47. Watkins CJCH (1989) Learning from delayed rewards. PhD thesis, King's College, Cambridge

48. Williams RJ (1992) Simple statistical gradient-following algorithms for connectionist reinforcement learning. Mach Learn 8(3):229-256. https://doi.org/10.1007/BF00992696

49. Yang LC, Chou SY, Yang YH (2017) MidiNet: a convolutional generative adversarial network for symbolic-domain music generation. arXiv e-prints arXiv: 1703.10847

Publisher's Note Springer Nature remains neutral with regard to jurisdictional claims in published maps and institutional affiliations. 